\title{
Nervios Digitales Palmares Comunes del Ramo Superficial del Nervio Ulnar y Nervios Digitales Plantares Comunes del Ramo Superficial del Nervio Plantar Lateral. Un Error Anatómico en Terminologia Anatomica
}

\author{
Common Palmar Digital Nerves from the Superficial Branch of the Ulnar Nerve and Common \\ Plantar Digital Nerves from Superficial Branch of the Lateral Nerve. An Anatomical Error in \\ Terminologia Anatomica
}

Mariano del Sol ${ }^{*, * *} \&$ Bélgica Vásquez $z^{* * * * * * * * *}$

DEL SOL, M. \& VÁSQUEZ, B. Nervios digitales palmares comunes del ramo superficial del nervio ulnar y nervios digitales plantares comunes del ramo superficial del nervio plantar lateral. Un error anatómico en Terminologia Anatomica. Int. J. Morphol., 32(3):10601063, 2014.

RESUMEN: Terminologia Anatomica contempla la existencia de nervios digitales palmares comunes del ramo superficial del nervio ulnar y nervios digitales plantares comunes del ramo superficial del nervio plantar lateral. De acuerdo a la mayoría de los textos clásicos y estudios anatómicos de las regiones de la palma de la mano y de la planta del pie, solo existiría un nervio digital palmar común (IV) originado del ramo superficial del nervio ulnar y un nervio digital plantar común (IV) con origen en el ramo superficial del nervio plantar lateral. Realizamos una revisión anatómica de la inervación cutánea de la palma de la mano observando el comportamiento de los nervios mediano y ulnar y de la planta del pie a través de los nervios plantares medial y lateral, comentamos la literatura relacionada y concluimos que debe corregirse la Terminologia Anatomica, en el término A14.2.03.046 (Nn. digitales palmares comunes) y en el término A14.2.07.071 (Nn. digitales plantares comunes), los cuales deberían denominarse N. digital palmar común y N. digital plantar común, ambos corresponderían al IV nervio digital palmar común y IV nervio digital plantar común, respectivamente.

PALABRAS CLAVE: Nervio ulnar; Nervios digitales palmares; Nervio plantar lateral; Nervios digitales plantares.

\section{INTRODUCCIÓN}

Un resumen bastante suscinto y preciso de la inervación cutánea de la palma de la mano la expresan Testut \& Jabob (1979) quienen señalaron tres territorios sensitivos a saber: 1. Por medial, el territorio del nervio ulnar, que ocupa la región hipotenar; 2. por lateral y proximal, en la parte superolateral de la región tenar, un territorio de dimensiones variables, inervado por los nervios musculocutáneo y radial; 3. en la parte mediana y en la inferior, el territorio del nervio mediano. Sin embargo, Testut \& Latarjet (1969) señalaron que en la cara palmar de la mano existen únicamente dos territorios: el del nervio mediano por lateral y el del nervio ulnar por medial.

De acuerdo a lo anterior y examinando la inervación sensitiva de la palma de la mano y de los dedos, ella está dada por los nervios digitales palmares comunes y nervios digitales palmares propios. Sin embargo, sería solamente un nervio digital palmar común proveniente del ramo superficial del nervio ulnar quien contribuiría a la inervación de la mano, ocurriendo lo mismo en la planta del pie donde el ramo superficial del nervio plantar lateral otorgaría solamente un nervio digital plantar común (IV). En consecuencia Terminologia Anatomica estaría considerando erróneamente un mayor número de nervios digitales palmares comunes del nervio ulnar, como también un mayor número de nervios digitales plantares comunes del ramo superficial del nervio plantar lateral.

Una descripción de estos nervios nos permitirá esclarecer los términos anatómicos que debieran ser utilizados.

\footnotetext{
* Centro de Excelencia en Ciencias Morfológicas y Quirúrgicas - CEMyQ,Universidad de La Frontera, Chile.

** Centro de Investigación en Ciencias Biomédicas, Universidad Autónoma de Chile.

**** Doctorado en Ciencias Morfológicas, Universidad de La Frontera, Chile.

***** Universidad de Tarapacá, Chile.
} 
A continuación como aparecen mencionados estos nervios en Terminologia Anatomica.

\section{A14.2.03.031 N. Mediano \\ A14.2.03.038 Nn. digitales palmares comunes \\ A.14.2.03.039 Nn. digitales palmares propios \\ A14.2.03.040 N. ulnar \\ A14.2.03.045 Ramo superficial \\ A14.2.03.046 Nn. digitales palmares comunes \\ A14.2.03.047 Nn. digitales palmares propios}
A14.2.07.058 N. tibial
A14.2.07.066 N. plantar medial
A14.2.07.067 Nn. digitales plantares comunes
A14.2.07.068 Nn. digitales plantares propios
A14.2.07.069 N. plantar lateral
A14.2.07.070 R. superficial
A14.2.07.071 Nn. digitales plantares comunes
A14.2.07.072 Nn. digitales plantares propios

Para poder justificar el error de Terminologia Anatomica anteriormente señalado, describiremos brevemente la anatomía de la inervación sensitiva en la palma de la mano y en la planta del pie.

Nervio mediano. Frecuentemente a su entrada a la palma de la mano se divide en un ramo lateral que da origen a ramos digitales para el pulgar y para el lado lateral del dedo indicador (Primer nervio digital palmar común) y un ramo medial, que da ramos digitales para los lados adyacentes de los dedos indicador, medio y anular (segundo y tercer nervios digitales palmares comunes). Los ramos digitales palmares comunes pasan distalmente profundos al arco palmar superficial, al inicio son anteriores a los tendones de los músculos flexores largos de los dedos. Dos nervios digitales palmares propios destinados al pulgar, a veces se originan en un tronco común, el nervio que suple la parte lateral del pulgar cruza anteriormente al tendón del músculo flexor largo del pulgar. El nervio digital palmar propio que inerva la parte lateral del dedo índice también inerva el primer músculo lumbrical. Dos nervios digitales palmares comunes (II y III) pasan distalmente entre los tendones de los músculo flexores largos de los dedos. El nervio digital palmar común lateral se divide distalmente en la palma de la mano en dos nervios digitales palmares propios que atraviesan los lados adyacentes de los dedos índice y medio. El nervio digital palmar común medial (III) se divide en dos nervios digitales palmares propios que inervan los lados adyacentes de los dedos medio y anular. El nervio digital común lateral (II) inerva el segundo músculo lumbrical y el medial (III) puede inervar el tercer músculo lumbrical. Puede existir un ramo comunicante entre el III nervio digital palmar común, destinado a los dedos medio y anular y el IV nervio digital palmar co- mún, destinado a los dedos anular y mínimo que proviene del nervio ulnar.

Nervio ulnar. En la palma de la mano, el ramo superficial del nervio ulnar inerva el músculo palmar corto y la piel del lado medial de la mano. El ramo superficial del nervio ulnar se divide en dos nervios digitales palmares, uno de ellos es el nervio digital palmar propio que inerva el lado medial del dedo mínimo y el otro es el IV nervio digital palmar común el cual se divide en dos nervios digitales palmares propios destinados a la inervación de los lados adyacentes de los dedos mínimo y anular.

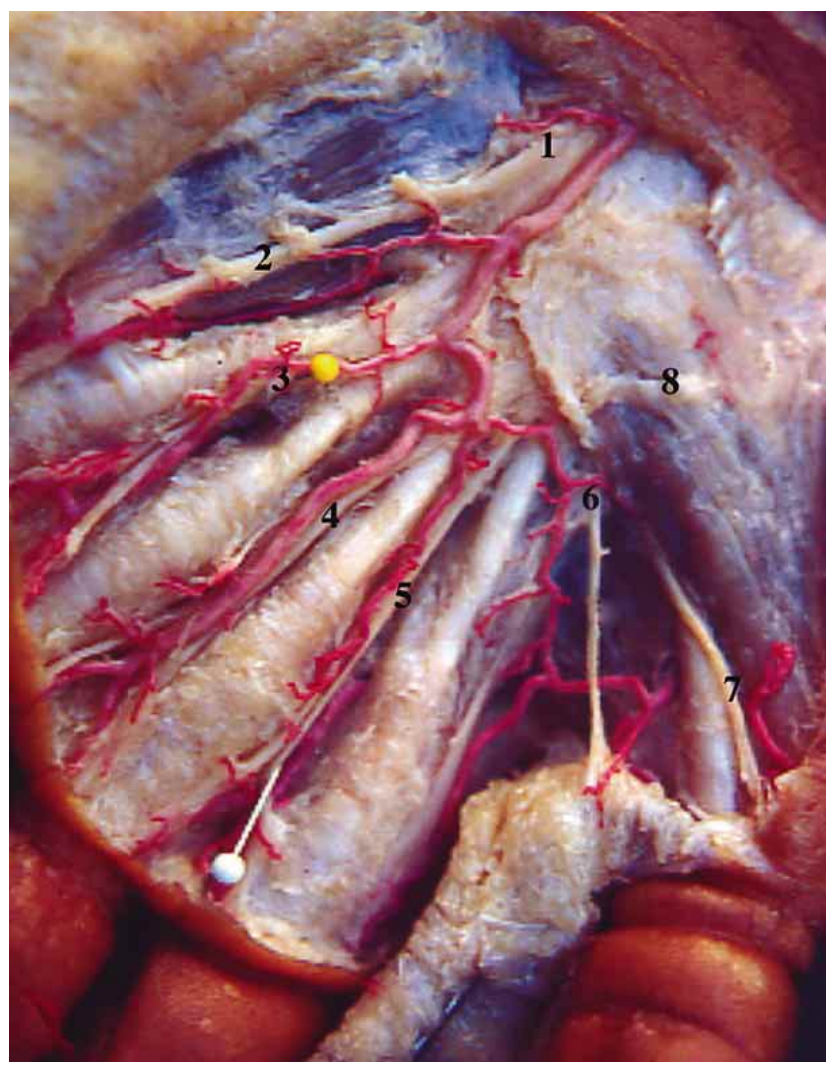

Fig. 2. Inervación de la palma de la mano. 1. Ramo superficial del nervio ulnar; 2. Nervio digital palmar propio del dedo mínimo (medial); 3. IV nervio digital palmar común del ramo superficial del nervio ulnar; 4,5,6. III,II y I nervios digitales palmares comunes del nervio mediano; 7. Nervio digital palmar propio lateral del pulgar, 8 . Ramo recurrente del nervio mediano (Gentileza Dr. Olave).

Con respecto a la inervación sensitiva de la planta del pie se realiza a través de los nervios plantares medial y lateral.

Nervio plantar medial. Diversos ramos cutáneos del nervio plantar medial atraviesan la aponeurosis plantar entre los músculos abductor del hálux y flexor corto de los dedos. Entre los músculos flexor corto del hálux y abductor del hálux, 
el nervio plantar medial da origen al nervio digital plantar propio destinado a la parte medial del hálux y luego se divide próximo a la base de los huesos metatarsianos en los nervios digitales plantares comunes I, II y III. Estos nervios pasan entre las proyecciones de la aponeurosis plantar que a este nivel es más delgada y cada uno de ellos se divide en dos nervios digitales plantares propios. El I nervio digital plantar común a través de sus nervios digitales plantares propios inerva las caras adyacentes del hálux y II dedo inervando además el I músculo lumbrical; el II nervio digital común se divide en los nervios digitales plantares propios que inervan las caras adyacentes del segundo y tercer dedo; el III nervio digital plantar común se divide también en dos nervios digitales plantares propios que inervan las caras adyacentes del tercer y cuarto dedos, pudiendo recibir eventualmente un ramo del ramo superficial del nervio plantar lateral.

Nervio plantar lateral. Un parte de la inervación sensitiva del nervio plantar lateral está destinada a la piel del dedo mínimo y la mitad lateral del cuarto dedo. Según Coronado \& del Sol ( 2010), la división del nervio plantar lateral en ramos superficial y profundo se produce aproximadamente entre los músculos flexor del dedo mínimo y abductor del dedo mínimo lateralmente y músculo flexor corto de los dedos medialmente (lateral al músculo cuadrado plantar). Se divide en dos ramos, uno lateral el nervio digital plantar propio del dedo mínimo que inerva su parte lateral y un ramo medial que corresponde al IV nervio digital plantar común, el cual se divide en dos nervios digitales plantares propios que inervan las caras adyacentes de los dedos cuarto y mínimo.

\section{DISCUSIÓN}

Los nervios digitales palmares comunes provenientes del nervio mediano tienen una distribución similar a los nervios digitales plantares comunes del nervio plantar medial. Asimismo, la distribución de los ramos palmares común y propios derivados del ramo superficial del nervio ulnar y los ramos plantares común y propios del ramo superficial del nervio plantar lateral presentan una distribución muy semejante en cuanto a su inervación cutánea a nivel digital.

Como lo indica la literatura y la experiencia personal, el límite de separación de los territorios del nervio mediano y del nervio ulnar está bien determinado por una línea ligeramente oblicua que, partiendo del centro de la muñeca, llega hasta el extremo libre del dedo anular, siguiendo la línea axil de este dedo (Testut \& Latarjet, Testut \& Jacob).

Con algunas pequeñas variaciones terminológicas y también anatómicas, en general los distintos autores descri- ben que el ramo superficial del nevio ulnar emite un ramo para el músculo palmar corto y se divide en el IV nervio digital palmar común y digital palmar propio para la cara medial del dedo mínimo (Pacheco \& Olave, 2009; Pró, 2014)

Torrez \& Olave (2008), señalaron que los nervios digitales palmares comunes del nervio mediano contenían más fibras que el IV nervio digital palmar común del ramo superficial del nervio ulnar, seguramente por que este último entregaba, vía ramo comunicante, parte de sus fibras para completar el III nervio digital palmar común. Similar observación fue realizada por Olave et al. (1998) entre los nervios digitales palmares comunes del III y IV espacios interóseos, el primero del nervio mediano y el segundo del nervio ulnar, debido a la existencia de un ramo que los comunica.

Según Williams et al. (1995) el ramo superficial del nervio ulnar se divide en dos nervios digitales palmares, que pueden ser palpados contra el gancho del hueso uncinado, uno de éstos inerva el lado medial del dedo mínimo, el otro (un nervio digital palmar común) envía un un pequeño ramo para el nervio mediano y se divide en dos nervios digitales palmares propios para los lados adyacentes de los dedos mínimo y anular. Sin embargo, en el pie estos autores señalan que el ramo superficial del nervio plantar lateral se divide en dos nervios digitales plantares comunes: el lateral suple el lado lateral del dedo mínimo, el músculo flexor corto del dedo mínimo y los dos músculos interóseos situados en el IV espacio intermetatarsiano; el medial se comunica con el III nervio digital plantar común del nervio plantar medial, dividiéndose en dos para suplir los lados adyacentes del IV y $\mathrm{V}$ dedos.

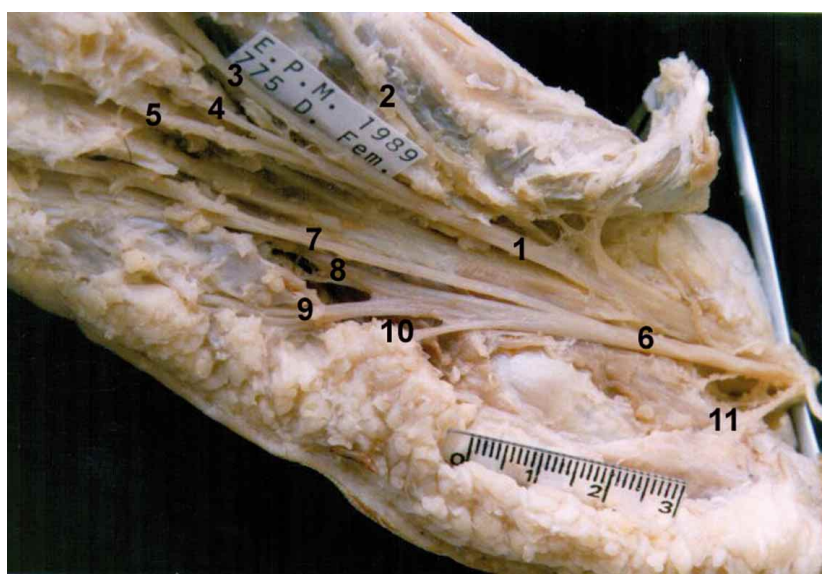

Fig. 2. Inervación de la planta del pie. Nervio plantar medial; 2. Nervio digital plantar propio del hálux (medial); 3,4,5. Nervios digitales plantares I, II y III; 6. Nervio plantar lateral; 7. IV Nervio digital plantar común; 8. Ramo profundo del nervio plantar lateral; 9. Nervio digital plantar propio (lateral) para el dedo mínimo; 10. Nervio cutáneo lateral; 11. Nervio para el músculo abductor del dedo mínimo. (del Sol \& Prates). 
La distribución sensitiva del nervio ulnar no está exenta de variaciones como lo señalaron Ferrari \& Gilbert (1991) y Pacheco \& Olave, respecto al aumento de su territorio de inervación, llegando a inervar parte del dedo medio, no es lo normal que ocurre en la palma de la mano.

Con respecto al nervio plantar lateral del Sol \& Prates (1991) en 116 pies determinaron que el ramo superficial del nervio plantar lateral después de un corto trayecto se dividía en dos ramos, siendo sus componentes dos nervios digitales plantares: común (IV) y propio del dedo mínimo. Variaciones diversas fueron observadas principalmente en relación a los componentes de los ramos superficial y profundo.
En resumen la inervación cutánea en la planta del pie está dada por delante del talón por los nervios plantares medial y lateral, cuyo límite de separación del territorio de estos dos nervios está representado, por una línea oblicua que parte del margen medial del pie, en el punto de unión de su tercio posterior con sus dos tercios anteriores y termina en el extremo libre del cuarto dedo, siguiendo el eje de éste.

En conclusión, el ramo superficial del nervio ulnar otorga solamente un nervio digital palmar común (IV) y el ramo superficial del nervio plantar lateral también solo un nervio digital plantar común (IV), lo que debe ser corregido en Terminologia anatomica.

DEL SOL, M. \& VÁSQUEZ, B. Common palmar digital nerves from the superficial branch of the ulnar nerve and common plantar digital nerves from the superficial branch of the lateral plantar nerve. An Anatomical error in Terminologia Anatomica. Int. J. Morphol., 32(3):1060-1063, 2014.

SUMMARY: Terminologia Anatomica contemplates the existence of common palmar digital nerves from the superficial branch of the ulnar nerve and common plantar digital nerves from the superficial branch of the lateral plantar nerve. According to most classical texts and anatomical studies of the regions palmar and plantar, there would only be common palmar digital nerve (IV) originated from the superficial branch of the ulnar nerve and common plantar digital nerve (IV) arising from the superficial branch of lateral plantar nerve. We conducted an anatomical review of the cutaneous innervation of the palm observing the behavior of the median and ulnar nerves, and the foot through the medial and lateral plantar nerves. In this study we discuss the related literature and conclude that Terminologia Anatomica must be corrected in the A14.2.03.046 term (Nn. common palmar digital) and in A14.2.07.071 term (Nn. common plantar digital ), which should be called: common palmar digital nerve and common plantar digital nerve, corresponding to both the IV common palmar digital nerve and IV common plantar digital nerve, respectively.

KEYWORDS: Ulnar nerve; Palmar digital nerves; Lateral plantar nerve; Plantar digital nerves.

\section{REFERENCIAS BIBLIOGRÁFICAS}

Coronado, J. C. \& del Sol, M. Inervación de los músculos interóseos plantares del pie humano. Int. J. Morphol., 28(4):999-1002, 2010.

del Sol, M. \& Prates, J. C. Ramos componentes de la división del nervio plantarlateral en el Hmbre. Rev. Chil. Anat., 9(2):197-11, 1991.

Ferrari, G. P. \& Gilbert, T. A. The superficial anastomosis on the palm of the hand between the ulnar and median nerves. J. Hand Surg., 16(5):511-4, 1991.

Pacheco, J. P. \& Olave, E. Contribución proximal del nervio ulnar para la formación del nervio digital palmar propio ulnar del dedo mínimo. Int. J. Morphol., 27(4):1169-72, 2009.

Olave, E.; del Sol, M. \& Gabrielli, C. Disposición y relaciones del ramo comunicante entre los nervios ulnar y mediano en la mano humana. Rev. Chil. Anat., 16(2):263-9, 1998.

Pró, E. A. Anatomía clínica. 2 ed. Buenos Aires, Panamericana, 2014.

Testut, L. \& Jacob, O. Tratado de anatomía topográfica con aplicaciones medicoquirúrgicas. 8. ed. Barcelona, Salvat, 1979. V. 2.
Testut, L. \& Latarjet, A. Tratado de anatomía humana. 9. ed. Barcelona, Salvat, 1969. V. 3.

Torrez, J. C. \& Olave, E. Parámetros biométricos y morfometría de la porción terminal del nervio mediano, ramo superficial del nervio ulnar y nervios digitales palmares comunes de la mano humana. Int. J. Morphol., 26(3):675-9, 2008.

Williams, P. L.; Warwick, R.; Dyson, M. \& Bannister, L. H. Gray Anatomia. 37. ed. Rio de Janeiro, Guanabara Koogan, 1995. V. 2 .

Dirección para correspondencia:

Prof. Dr. Mariano del Sol

Facultad de Medicina

CEMyQ

Universidad de La Frontera

Temuco - CHILE

Email: mariano.delsol@ufrontera.cl

Recibido : 22-05-2014

Aceptado:18-07-2014 